\title{
Macroprolactinemia: Diagnostic, Clinical, and Pathogenic Significance
}

\author{
Akira Shimatsu' ${ }^{1}$ and Naoki Hattori ${ }^{2}$ \\ ${ }^{1}$ Clinical Research Institute, National Hospital Organization Kyoto Medical Center, 1-1 Mukaihata-cho, Fukakusa, \\ Fushimi-ku, Kyoto 612-8555, Japan \\ ${ }^{2}$ Department of Pharmaceutical Sciences, Ritsumeikan University, Shiga 525-8577, Japan
}

Correspondence should be addressed to Akira Shimatsu, ashimats@kyotolan.hosp.go.jp

Received 12 September 2012; Revised 2 November 2012; Accepted 8 November 2012

Academic Editor: Xavier Bossuyt

Copyright (C) 2012 A. Shimatsu and N. Hattori. This is an open access article distributed under the Creative Commons Attribution License, which permits unrestricted use, distribution, and reproduction in any medium, provided the original work is properly cited.

\begin{abstract}
Macroprolactinemia is characterized by a large molecular mass of PRL (macroprolactin) as the main molecular form of PRL in sera, the frequent elevation of serum PRL (hyperprolactinemia), and the lack of symptoms. Macroprolactin is largely a complex of PRL with immunoglobulin G (IgG), especially anti-PRL autoantibodies. The prevalence of macroprolactinemia is $10-25 \%$ in patients with hyperprolactinemia and $3.7 \%$ in general population. There is no gender difference and a long-term followup demonstrates that macroprolactinemia develops before middle age and is likely a chronic condition. Polyethylene-glycol- (PEG-) precipitation method is widely used for screening macroprolactinemia, and gel filtration chromatography, protein $\mathrm{A} / \mathrm{G}$ column, and ${ }^{125} \mathrm{I}-\mathrm{PRL}$ binding studies are performed to confirm and clarify its nature. The cross-reactivity of macroprolactin varies widely according to the immunoassay systems. The epitope on PRL molecule recognized by the autoantibodies is located close to the binding site for PRL receptors, which may explain that macroprolactin has a lower biological activity. Hyperprolactinemia frequently seen in macroprolactinemic patients is due to the delayed clearance of autoantibody-bound PRL. When rats are immunized with rat pituitary PRL, anti-PRL autoantibodies are produced and hyperprolactinemia develops, mimicking macroprolactinemia in humans. Screening of macroprolactinemia is important for the differential diagnosis of hyperprolactinemia to avoid unnecessary examinations and treatments.
\end{abstract}

\section{Introduction}

Prolactin (PRL) is an anterior pituitary hormone that plays an important role in lactation during pregnancy but has many other biological functions such as osmoregulation, angiogenesis, and immunoregulation [1]. PRL facilitates the maturation of $\mathrm{T}$ cells via IL-2 receptor expression, impairs $B$ cell tolerance to self-antigens through the anti-apoptotic effect, develops antigen-presenting cells, and enhances immunoglobulin production [2]. The increase in serum PRL concentrations (hyperprolactinemia) often develops symptoms such as amenorrhea and galactorrhea in women and impotence in men. It is caused physiologically by pregnancy and pathologically by PRL secreting pituitary adenoma (prolactinoma), hypothalamic and pituitary diseases compressing pituitary stalk, antidopaminergic drugs, hypothyroidism, chest wall diseases, and hepatorenal disorders [3]. However, $29 \%$ of hyperprolactinemia has been classified as "idiopathic" because the causes are unknown [4]. Microadenomas in the pituitary gland that cannot be detected by computed tomography (CT) or magnetic resonance imaging (MRI) have been postulated to enter this category.

Anti-PRL autoantibody was found to be one of the major causes of "idiopathic" hyperprolactinemia [5]. It binds to PRL (molecular mass of $23 \mathrm{kDa}$ ) forming a large immune complex of PRL with IgG (macroprolactin) and tends to increase serum PRL concentrations. Macroprolactinemia is defined as having macroprolactin (molecular mass greater than $150 \mathrm{kDa}$ ) in the predominant molecular form of PRL in sera.

There are reportedly several autoantibodies against hormones other than PRL: insulin [6], glucagon [7], thyroid 
hormone [8], parathyroid hormone [9], anterior pituitary hormones such as adrenocorticotropic hormone (ACTH) [10], luteinizing hormone (LH), follicle stimulating hormone (FSH) [11], growth hormone (GH) [12] and thyroid stimulating hormone (TSH) [13], and posterior pituitary hormones such as vasopressin and oxytocin [14]. This paper focuses on the diagnostic, clinical, and pathogenic features of macroprolactinemia.

\section{Diagnosis of Macroprolactinemia}

Macroprolactin is mostly a complex of PRL with $\operatorname{IgG}$, especially anti-PRL autoantibodies (Figure 1). The screening of macroprolactinemia is performed by polyethyleneglycol- (PEG-) precipitation method, and the confirmative and qualitative examinations include gel chromatography, protein A/G column, and ${ }^{125} \mathrm{I}$-PRL binding studies [15]. Advantage and disadvantage of these methods are summarized in Table 1.

2.1. Polyethylene-Glycol- (PEG-) Precipitation. Since we first applied PEG precipitation method, which had been used to detect anti-insulin autoantibodies, to diagnose macroprolactinemia due to anti-PRL autoantibodies [5], this method has been used for the screening of macroprolactinemia because of its simplicity [19-28]. The method was validated against gel filtration chromatography, a gold standard for the diagnosis of macroprolactinemia [19]. To determine free PRL concentrations, serum samples $(50 \mu \mathrm{L})$ are mixed vigorously with $50 \mu \mathrm{L}$ of cold PEG (molecular weight 6000 , 25\% in water) and centrifuged at $9,100 \times \mathrm{g}$ for $10 \mathrm{~min}$ to remove macroprolactin. Serum samples are treated identically, but with water instead of PEG, to determine total PRL concentrations. The PEG-precipitable PRL (\%), which represents the amount of macroprolactin, is calculated as follows: (total PRL-free PRL)/total PRL $\times 100$. PEG-precipitation ratio greater than $60 \%$ (recovery less than $40 \%$ ) is used as the cutoff value for the diagnosis of macroprolactinaemia. Gibney et al. [23] proposed an alternative presentation, that is, showing absolute values of free PRL in the supernatant after PEG precipitation. When PRL levels after PEG precipitation fall within a reference range derived from similarly treated normal sera, this is considered a normal result. From a clinical point of view, this is reasonable because such presentation can disclose the patients who need further examinations and treatments for hyperprolactinemia. Both presentations (PEG-precipitation ratio and free PRL value) may be desirable until the time when it is clarified that antiPRL autoantibodies in macroprolactinemia do not affect PRL actions and macroprolactinemia is totally a benign condition.

As to the prevalence of macroprolactinemia screened by PEG-precipitation method, it is noteworthy that the detectability of macroprolactin varies a lot according to the PRL assay systems [28]. It is possible that some reagent antibodies against PRL in assay kits can recognize anti-PRL autoantibody-bound PRL and others not. Moreover, it may

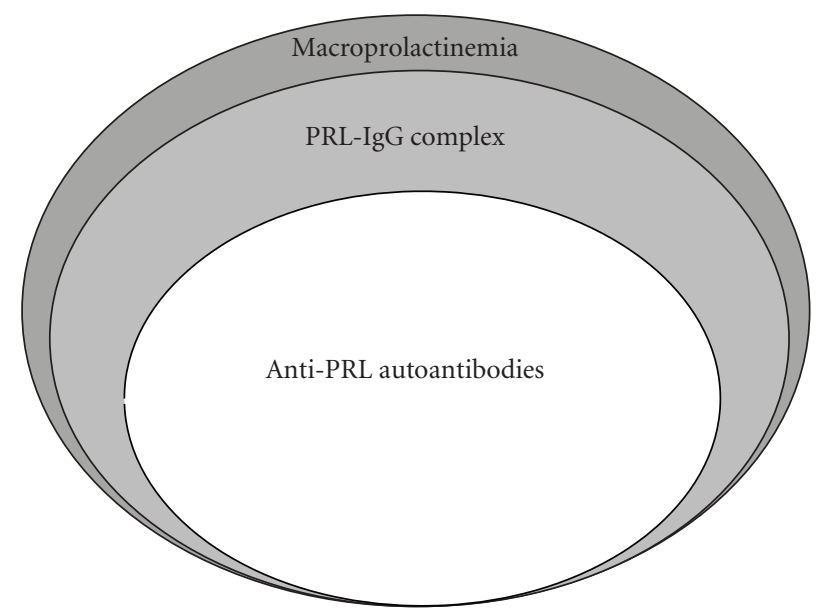

FIgure 1: Macroprolactinemia, IgG-bound PRL, and anti-PRL autoantibodies. Macroprolactinemia is a heterogeneous condition with different etiologies; $87 \%$ of macroprolactin was PRL-IgG complex and $67 \%$ of macroprolactin was autoantibody-bound PRL [15]. Although anti-PRL autoantibody-bound PRL is a major form of PRL-IgG complex and PRL-IgG complex is a major form of macroprolactin, there may be PRL-IgG complex other than autoantibodies and macroprolactin other than PRL-IgG complex as shown in grey area. The diagnosis of macroprolactinemia is made based on the results of PEG-precipitation method or gel filtration chromatography, IgG-bound PRL by protein A or protein G column method, and anti-PRL autoantibody-bound PRL by ${ }^{125} \mathrm{I}-$ PRL binding study.

be attributable to the heterogeneity of macroprolactinemia $[17,29]$.

2.2. Gel Filtration Chromatography. Traditionally, gel filtration chromatography has been used to separate various molecular forms of PRL: little (monomeric) PRL (molecular size: $23 \mathrm{kDa})$, big PRL $(45-50 \mathrm{kDa})$, and big-big PRL (more than $100 \mathrm{kDa}$ ) [30]. Little PRL is a major form of pituitary and serum PRL, big PRL is a dimer of little (monomeric) PRL, and big-big PRL has been believed to be an aggregate of monomeric PRL. Macroprolactin is defined as a large molecular-sized PRL greater than $100 \mathrm{kDa}$ that is included in big-big PRL, and the state in which the ratio of macroprolactin is substantially increased in sera is called macroprolactinemia (Figure 2). There is no a clear-cut value of "substantial increase", but conventionally a diagnosis of macroprolactinemia is made when more than $30-60 \%$ of PRL is in the macroprolactin form of gel filtration chromatography [27]. Although gel filtration chromatography was regarded as the gold standard for the diagnosis of macroprolactinemia, it is time, labor, and cost consuming. Thus this method is used to confirm the diagnosis of macroprolactinemia.

2.3. Protein A/G Column. Protein A binds to the Fc portion of immunoglobulin molecules without interfering with the antigen-binding site. Protein G binds only to IgG and its subclasses, separating out $\operatorname{IgA}, \operatorname{IgM}, \operatorname{IgD}$, and albumin, 
TABLE 1: Advantage and disadvantage of methods for the diagnosis of macroprolactinemia.

\begin{tabular}{lll}
\hline & Advantage & Disadvantage \\
\hline Polyethylene glycol (PEG) & $\begin{array}{l}\text { Simple and inexpensive } \\
\text { Screening for macroprolactinemia }\end{array}$ & Not highly specific \\
\hline Gel chromatography & Accurate Confirming macroprolactinemia & Time consuming and expensive \\
\hline Protein A/G & $\begin{array}{l}\text { Identifying IgG-bound PRL } \\
\text { (common cause of macroprolactin) }\end{array}$ & Expensive \\
\hline${ }^{125}$ I-PRL binding & $\begin{array}{l}\text { Identifying anti-PRL autoantibodies } \\
\text { (common cause of IgG-bound PRL) }\end{array}$ & $\begin{array}{l}\text { Time consuming and hazardous } \\
\text { Needs radio isotope facilities }\end{array}$ \\
\hline
\end{tabular}

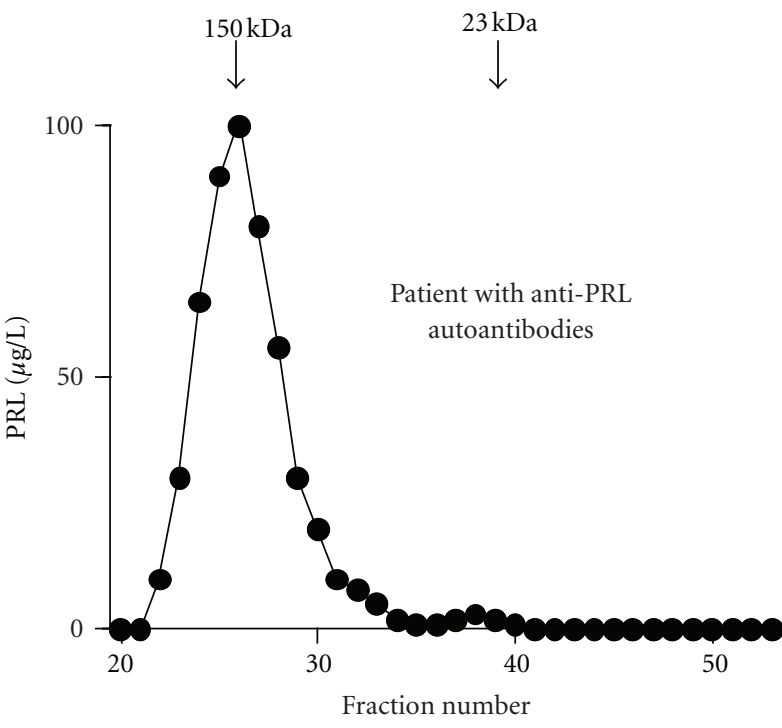

(a)

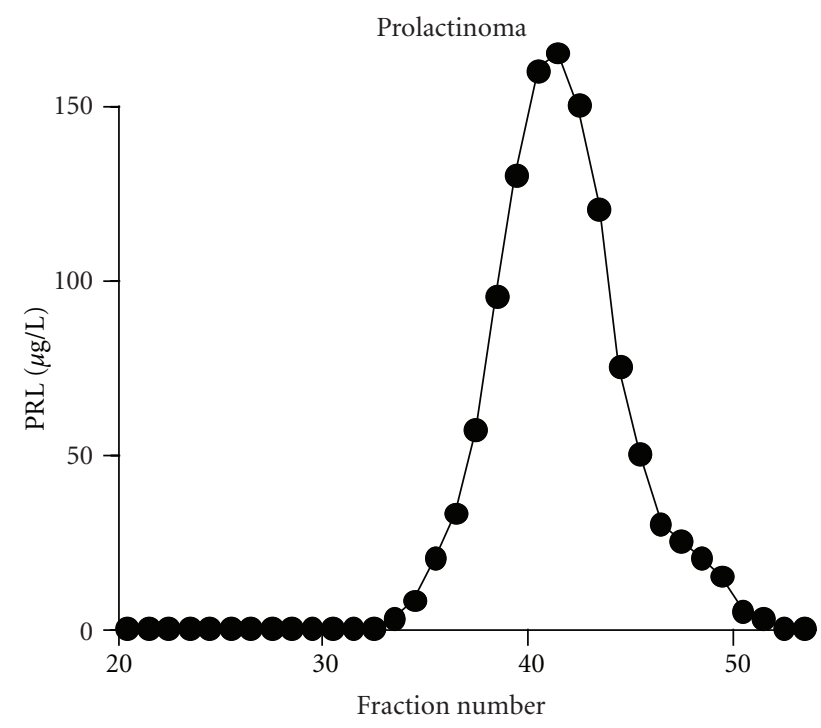

(b)

FIGURE 2: Gel filtration chromatography of macroprolactin and prolactin. Representative gel filtration chromatography of PRL in serum samples of macroprolactinemia (a) and prolactinoma (b). (Reproduced from [16]).

which may bind to protein A [31]. These reagents are used to identify macroprolactin due to PRL-IgG complexes, and most patients with macroprolactinemia belong to this category $[15,17]$.

2.4. ${ }^{125}$ I-PRL Binding Study. Serum samples $(100 \mu \mathrm{L})$ and ${ }^{125}$ I-PRL $(20000 \mathrm{cpm} / 50 \mu \mathrm{L}$ sodium phosphate buffer containing $0.1 \%$ bovine serum albumin and $0.1 \mathrm{~mol} / \mathrm{L} \mathrm{NaCl}$ ) were incubated for $1 \mathrm{~h}$ at room temperature. After incubation, $200 \mu \mathrm{L}$ of cold 25\% (wt/wt) PEG 6000 (final concentration $12.5 \%$ ) was added, and the mixture was mixed vigorously and centrifuged at $3000 \mathrm{rpm}$ for $20 \mathrm{~min}$. The sediment was washed once with $12.5 \%$ PEG, and the radioactivity was measured with $\gamma$-counter $[5,15,17]$.

\section{Clinical Significance}

3.1. Prevalence of Macroprolactinemia. Figure 3 shows the prevalence of macroprolactinemia and its gender and age dependency in a large group of hospital workers $(n=1330)$ [17]. Macroprolactinemia is present in $3.68 \%$ in general population. The prevalence is not different between women and men, and it tends to increase in elderly people. In patients with hyperprolactinemia, the prevalence of macroprolactinemia is reportedly $10-25 \%$ [20-23].

3.2. Symptoms. Hyperprolactinemia tends to develop in patients with macroprolactinemia [17] because of the delayed clearance of macroprolactin. Although hyperprolactinemia frequently causes menstrual irregularities and galactorrhea in women and loss of libido in men, patients with macroprolactinemia often lack such clinical symptoms of hyperprolactinemia [18]. In vitro experiment using T47D human breast cancer cells, the bioactivity of autoantibodybound PRL was found to be low [32], compatible with the clinical characteristics. However, controversial results are also reported [22] probably because the prevalence of macroprolactinemia is so high that the patients with macroprolactinemia might have other causes of hyperprolactinemia as well [27]. Women with macroprolactinemia can get pregnant and deliver normal babies without any treatments of hyperprolactinemia [33]. Long-term followup studies of macroprolactinemia show that macroprolactinemia persisted but no symptomatic progression was 


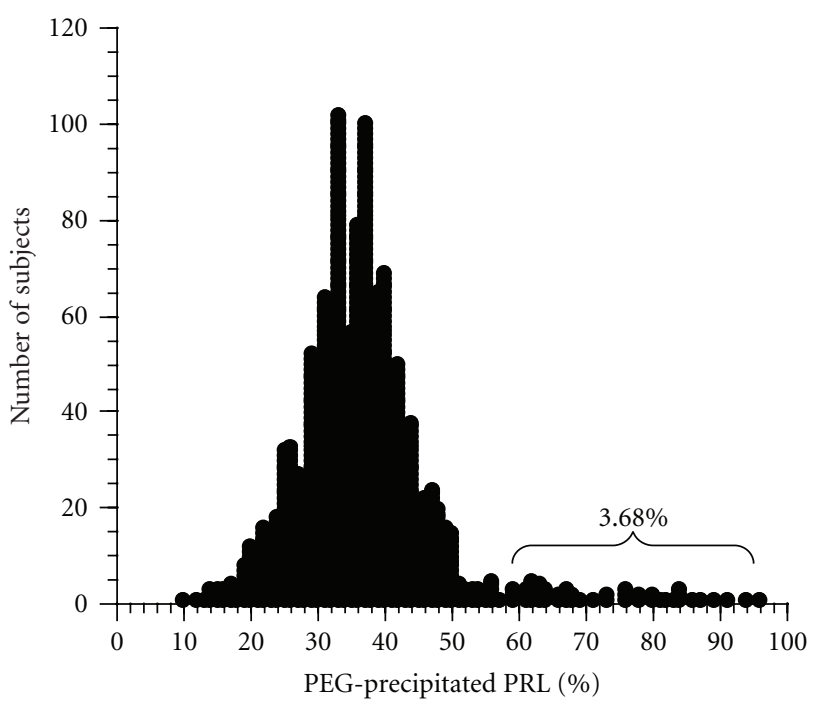

(a)

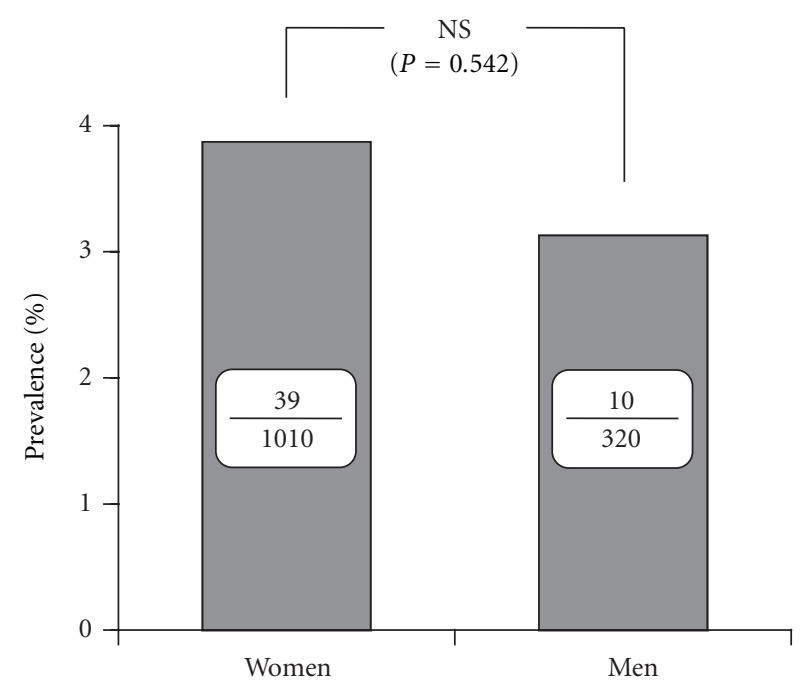

(b)

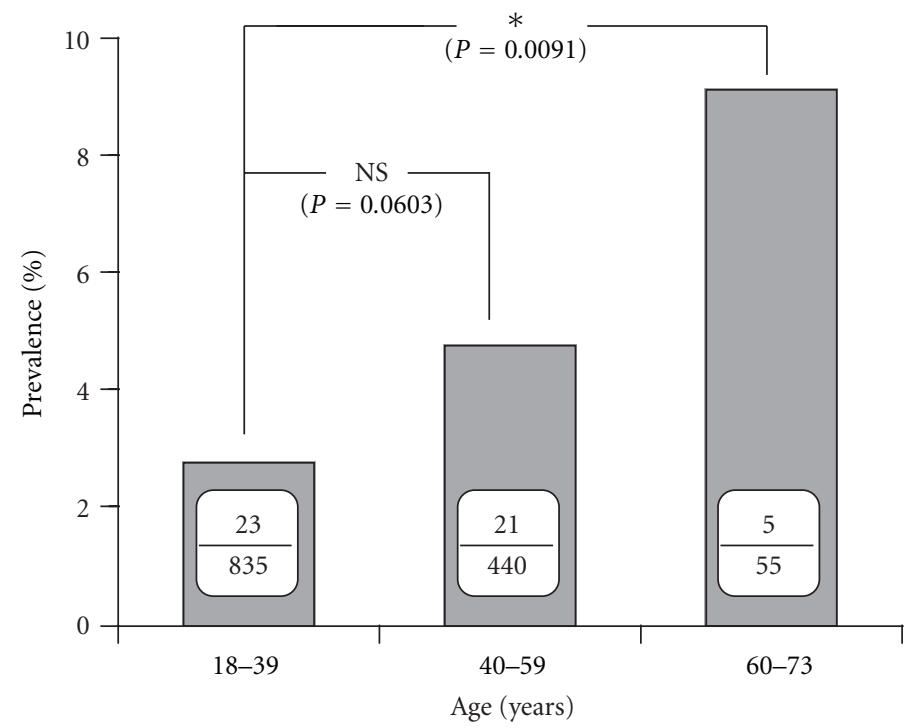

(c)

FIGURE 3: The prevalence of macroprolactinemia and its gender and age dependency in normal subjects. (a) The circle represents the value of PEG-precipitation ratio in each individual and the vertical line shows the number of subjects. The graph shows almost a normal distribution except for a subgroup (macroprolactinemia) having the ratio greater than $60 \%$ (mean $+2 \mathrm{SD}$ ). (b) The prevalence of macroprolactinemia in women and men. (c) The prevalence of macroprolactinemia in different age groups. (Reproduced from [17]).

noted $[15,34]$. Thus it is strongly recommended that all patients with hyperprolactinemia should take the screening test of macroprolactinemia in order to avoid unnecessary examinations and treatments.

3.3. Association with Other Autoimmune Disorders. AntiPRL autoantibodies are a major cause of macroprolactinemia. Thus it is possible that some autoimmune disorders might be accompanied with macroprolactinemia. There were some case reports showing the association between macroprolactinemia and autoimmune thyroid disorders such as Graves' disease and Hashimoto's thyroiditis $[35,36]$. Hyperprolactinemic SLE patients reportedly had a higher frequency of macroprolactinemia (14/43, 32.6\%) [37]. However, other studies examining a large number of patients revealed no specific association between macroprolactinemia and autoimmune disorders [22, 38]. It is likely that autoimmune mechanisms may be directed mainly toward prolactin molecule in macroprolactinemia.

\section{Pathogenic Significance}

4.1. Characteristics of Anti-PRL Autoantibodies. When IgG was purified from sera using protein $\mathrm{G}$ column, a significant amount of PRL was copurified with IgG in patients with macroprolactinemia. When this IgG fraction was analyzed 


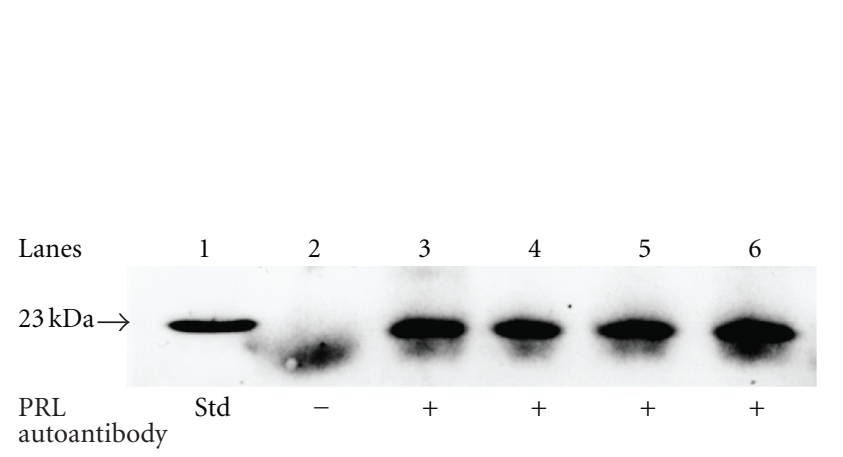

(a)

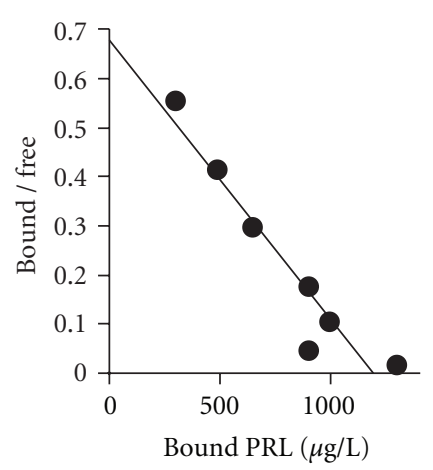

(b)

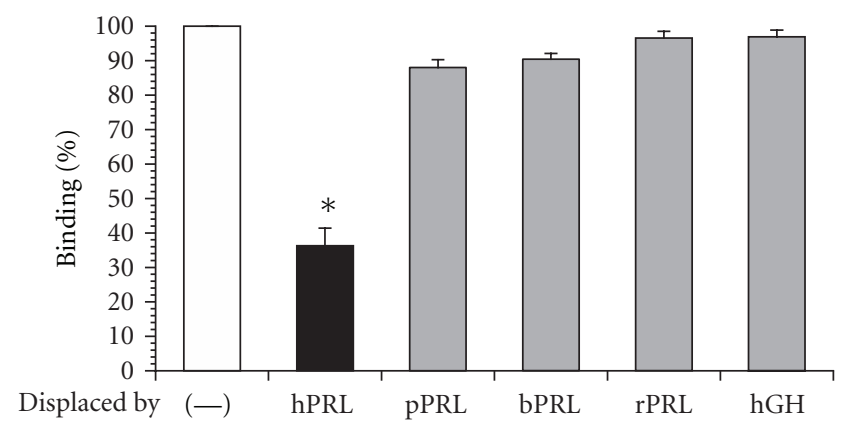

(c)

FIGURE 4: Electrophoresis of macroprolactin and Scatchard analysis of anti-PRL autoantibodies. (a) IgG was purified from the sera of the patients with macroprolactinemia (lanes 3-6) using a protein G column and run on SDS-PAGE under a nonreducing condition. PRL, which bound to the autoantibodies, was dissociated and immunostained at the same position as the $23 \mathrm{kDa}$ human PRL standard (lane 1 ). The $23 \mathrm{kDa}$ PRL band was not observed when IgG from a patient with prolactinoma was used (lane 2). The fuzzy bands migrating faster than PRL may be nonspecific staining of IgG light chain. (b) Scatchard analysis revealed low-affinity and high-capacity autoantibodies. (c) Displacement of ${ }^{125}$ I-hPRL-autoantibody complex by human PRL and other related peptides; hPRL: human PRL, pPRL: porcine PRL, bPRL: bovine PRL, rPRL:r at PRL, and hGH: human GH. Only hPRL could displace the binding of ${ }^{125}$ I-PRL with the autoantibodies. (Reproduced from $[5,15,18])$.

on SDS-PAGE under nonreducing condition, the IgGbound PRL was dissociated and immunostained at the same position as the $23 \mathrm{kDa}$ human PRL standard as shown in Figure 4(a), suggesting that PRL is noncovalently bound to IgG [18]. Scatchard analysis revealed low-affinity and highcapacity autoantibodies as shown in Figure $4(\mathrm{~b})[5,33]$ and only human PRL could displace the binding of ${ }^{125}$ I-PRL and anti-PRL autoantibodies as shown in Figure 4(c) $[15,39]$, suggestive of the specificity of the autoantibody to human PRL. The subclass of anti-PRL autoantibodies was mainly IgG4, suggesting that chronic antigen stimulation may be involved [40].

4.2. Mechanisms of Hyperprolactinemia. Dopamine or bromocriptine, dopamine $\mathrm{D}_{2}$ receptor agonist, decreases serum PRL concentrations [1]. Administration of these drugs to the patients with macroprolactinemia did not decrease serum PRL concentrations so much as in prolactinoma, suggesting that the clearance of PRL is delayed [33]. This was confirmed by the animal experiments showing that anti-PRL autoantibody-bound PRL was cleared from the rat circulation more slowly than monomeric PRL [18] and that hyperprolactinemia developed in animal model of macroprolactinemia [41]. Moreover, a significant positive correlation was present between anti-PRL autoantibody titers and serum PRL concentrations in humans [33], suggesting that anti-PRL autoantibody is a cause of hyperprolactinemia. It is assumed that the hypothalamic negative feedback mechanism by autoantibody-bound PRL does not work because the complex cannot access to the hypothalamus and/or anti-PRL autoantibodies interfere with the binding of PRL to the receptor. Thus, serum PRL concentrations may increase until free PRL concentrations exceed normal PRL concentrations, when negative feedback mechanisms begin to operate to make free PRL levels down. Actually, free PRL concentrations in sera from most patients with macroprolactinemia are within normal range $[17,27]$.

4.3. Mechanisms of Low Bioactivity. Macroprolactinemia is characterized by a lack of clinical symptoms of hyperprolactinemia and several studies using different PRL bioassay systems showed that the bioactivity of macroprolactin is low [32, 42, 43]. The epitope mapping using deleted PRL fragments revealed that the binding sites of PRL molecule 
to anti-PRL autoantibodies reside in $\mathrm{N}$ and $\mathrm{C}$ terminals of PRL [39]. The core human PRL structure is made up of four major $\alpha$-helices. The $\alpha$-helix groups are in two antiparallel pairs, helix 1/helix 4 and helix 2 /helix 3, each pair being packed more closely together. Therefore, several N- and Cterminal amino acid residues are located closely in the threedimensional structure forming a part of binding site 1 to human PRL receptors [44]. Thus, anti-PRL autoantibodies and PRL receptors bind to the similar regions on PRL molecule, raising a possibility that the autoantibodies may compete the binding of PRL molecule to its receptors, resulting in the low biological activity.

4.4. Possible Causes of Anti-PRL Autoantibody Production. Mechanisms involved in the development of anti-PRL autoantibodies are unknown. Genetic susceptibility and environmental factors may alter the immune response in hosts as postulated in other autoimmune disorders [45]. On the other hand, some changes in PRL molecule may increase the antigenicity leading to the production of anti-PRL autoantibodies. The finding that IgG4 was a predominant subtype of anti-PRL autoantibodies supports the latter possibility because IgG4 response occurs in chronic antigen response. Switch from IgG1 to IgG4 response is driven by the repeated exposure to allergens, and IgG4 is a predominant subclass in other autoantibodies [46, 47]. Posttranslational modifications such as phosphorylation of PRL might be involved in the possible altered antigenicity [40].

\section{Conclusion}

Macroprolactinemia should be examined in all serum samples with hyperprolactinemia for the differential diagnosis because it is one of the major causes of hyperprolactinemia. Neither medications nor further examinations are recommended if free PRL concentrations after precipitating macroprolactin with PEG are normal, because the biological activity of macroprolactin is low and pregnancy is possible without any treatment for hyperprolactinemia. Macroprolactinemia is a heterogeneous condition with different etiologies and further study is necessary to glean it in its entirety.

\section{References}

[1] M. E. Freeman, B. Kanyicska, A. Lerant, and G. Nagy, "Prolactin: structure, function, and regulation of secretion," Physiological Reviews, vol. 80, pp. 1523-1631, 2000.

[2] S. Shelly, M. Boaz, and H. Orbach, "Prolactin and autoimmunity," Autoimmunity Reviews, vol. 11, no. 6-7, pp. A465-A470, 2012.

[3] M. E. Molitch, "Pathologic hyperprolactinemia," Endocrinology and Metabolism Clinics of North America, vol. 21, no. 4, pp. 877-901, 1992.

[4] K. Berinder, I. Stackenäs, O. Akre, A. L. Hirschberg, and A. L. Hulting, "Hyperprolactinaemia in 271 women: up to three decades of clinical follow-up," Clinical Endocrinology, vol. 63, no. 4, pp. 450-455, 2005.

[5] N. Hattori, T. Ishihara, K. Ikekubo, K. Moridera, M. Hino, and H. Kurahachi, "Autoantibody to human prolactin in patients with idiopathic hyperprolactinemia," Journal of Clinical Endocrinology and Metabolism, vol. 75, no. 5, pp. 12261229, 1992.

[6] H. B. Burch, S. Clement, M. S. Sokol, and F. Landry, "Reactive hypoglycemic coma due to insulin autoimmune syndrome: case report and literature review," The American Journal of Medicine, vol. 92, no. 6, pp. 681-685, 1992.

[7] T. Sanke, M. Kondo, Y. Moriyama, K. Nanjo, K. Iwo, and K. Miyamura, "Glucagon binding autoantibodies in a patient with hyperthyroidism treated with methimazole," Journal of Clinical Endocrinology and Metabolism, vol. 57, no. 6, pp. 1140-1144, 1983.

[8] S. Sakata, S. Nakamura, and K. Miura, "Autoantibodies against thyroid hormones or iodothyronine: implications in diagnosis, thyroid function, treatment, and pathogenesis," Annals of Internal Medicine, vol. 103, no. 4, pp. 579-589, 1985.

[9] B. Cavaco, V. Leite, M. M. Loureiro et al., "Spontaneously occurring anti-PTH autoantibodies must be considered in the differential diagnosis of patients with elevated serum PTH levels," Journal of Endocrinological Investigation, vol. 22, no. 11, pp. 829-834, 1999.

[10] R. Wheatland, "Chronic ACTH autoantibodies are a significant pathological factor in the disruption of the hypothalamicpituitary-adrenal axis in chronic fatigue syndrome, anorexia nervosa and major depression," Medical Hypotheses, vol. 65, no. 2, pp. 287-295, 2005.

[11] W. R. Meyer, G. Lavy, A. H. DeCherney, I. Visintin, K. Economy, and J. L. Luborsky, "Evidence of gonadal and gonadotropin antibodies in women with a suboptimal ovarian response to exogenous gonadotropin," Obstetrics and Gynecology, vol. 75, no. 5, pp. 795-799, 1990.

[12] T. J. Wilkin, L. Voss, A. Tuck, H. Bullen, and P. Betts, "Autoantibodies to endogenous growth hormone in short children (The Wessex Growth Study)," Autoimmunity, vol. 14, no. 1, pp. 67-72, 1992.

[13] J. H. Lazarus, R. John, and J. Ginsberg, "Transient neonatal hyperthyrotrophinaemia: a serum abnormality due to transplacentally acquired antibody to thyroid stimulating hormone," British Medical Journal, vol. 286, no. 6365, pp. 592$594,1983$.

[14] S. O. Fetissov, J. Harro, M. Jaanisk et al., "Autoantibodies against neuropeptides are associated with psychological traits in eating disorders," Proceedings of the National Academy of Sciences of the United States of America, vol. 102, no. 41, pp. 14865-14870, 2005.

[15] N. Hattori, T. Ishihara, Y. Saiki, and A. Shimatsu, "Macroprolactinaemia in patients with hyperprolactinaemia: composition of macroprolactin and stability during long-term followup," Clinical Endocrinology, vol. 73, no. 6, pp. 792-797, 2010.

[16] N. Hattori, K. Ikekubo, T. Ishihara, K. Moridera, M. Hino, and H. Kurahachi, "A normal ovulatory woman with hyperprolactinemia: presence of anti-prolactin autoantibody and the regulation of prolactin secretion," Acta Endocrinologica, vol. 126, no. 6, pp. 497-500, 1992.

[17] N. Hattori, T. Ishihara, and Y. Saiki, "Macroprolactinaemia: prevalence and aetiologies in a large group of hospital workers," Clinical Endocrinology, vol. 71, no. 5, pp. 702-708, 2009.

[18] N. Hattori and C. Inagaki, "Anti-prolactin (PRL) autoantibodies cause asymptomatic hyperprolactinemia: bioassay and clearance studies of PRL-immunoglobulin G complex," Journal of Clinical Endocrinology and Metabolism, vol. 82, no. 9, pp. 3107-3110, 1997. 
[19] J. G. H. Vieira, T. T. Tachibana, L. H. Obara, and R. M. B. Maciel, "Extensive experience and validation of polyethylene glycol precipitation as a screening method for macroprolactinemia," Clinical Chemistry, vol. 44, no. 8 I, pp. 1758-1759, 1998.

[20] M. N. Fahie-Wilson and S. G. Soule, "Macroprolactinaemia: contribution to hyperprolactinaemia in a district general hospital and evaluation of a screening test based on precipitation with polyethylene glycol," Annals of Clinical Biochemistry, vol. 34, no. 3, pp. 252-258, 1997.

[21] H. Leslie, C. H. Courtney, P. M. Bell et al., "Laboratory and clinical experience in 55 patients with macroprolactinemia identified by a simple polyethylene glycol precipitation method," Journal of Clinical Endocrinology and Metabolism, vol. 86, no. 6, pp. 2743-2746, 2001.

[22] S. Vallette-Kasic, I. Morange-Ramos, A. Selim et al., "Macroprolactinemia revisited: a study on 106 patients," Journal of Clinical Endocrinology and Metabolism, vol. 87, no. 2, pp. 581588, 2002.

[23] J. Gibney, T. P. Smith, and T. J. McKenna, "The impact on clinical practice of routine screening for macroprolactin," Journal of Clinical Endocrinology and Metabolism, vol. 90, no. 7, pp. 3927-3932, 2005.

[24] B. Cavaco, V. Leite, M. A. Santos, E. Arranhado, and L. G. Sobrinho, "Some forms of big big prolactin behave as a complex of monomeric prolactin with an immunoglobulin $\mathrm{G}$ in patients with macroprolactinemia or prolactinoma," Journal of Clinical Endocrinology and Metabolism, vol. 80, no. 8, pp. 2342-2346, 1995.

[25] J. De Schepper, J. Schiettecatte, B. Velkeniers et al., "Clinical and biological characterization of macroprolactinemia with and without prolactin-IgG complexes," European Journal of Endocrinology, vol. 149, no. 3, pp. 201-207, 2003.

[26] L. Kavanagh, T. J. McKenna, M. N. Fahie-Wilson, J. Gibney, and T. P. Smith, "Specificity and clinical utility of methods for the detection of macroprolactin," Clinical Chemistry, vol. 52, no. 7, pp. 1366-1372, 2006.

[27] J. Gibney, T. P. Smith, and T. J. McKenna, "Clinical relevance of macroprolactin," Clinical Endocrinology, vol. 62, no. 6, pp. 633-643, 2005.

[28] T. P. Smith, A. M. Suliman, M. N. Fahie-Wilson, and T. J. McKenna, "Gross variability in the detection of prolactin in sera containing big big prolactin (macroprolactin) by commercial immunoassays," Journal of Clinical Endocrinology and Metabolism, vol. 87, no. 12, pp. 5410-5415, 2002.

[29] N. Hattori, "The frequency of macroprolactinemia in pregnant women and the heterogeneity of its etiologies," Journal of Clinical Endocrinology and Metabolism, vol. 81, no. 2, pp. 586-590, 1996.

[30] H. K. Suh and A. G. Frantz, "Size heterogeneity of human prolactin in plasma and pituitary extracts," Journal of Clinical Endocrinology and Metabolism, vol. 39, no. 5, pp. 928-935, 1974.

[31] B. Akerstrom and L. Bjorck, "A physicochemical study of protein $\mathrm{G}$, a molecule with unique immunoglobulin G-binding properties," Journal of Biological Chemistry, vol. 261, no. 22, pp. 10240-10247, 1986.

[32] N. Hattori, Y. Nakayama, K. Kitagawa, T. Ishihara, Y. Saiki, and C. Inagaki, "Anti-prolactin (PRL) autoantibodies suppress PRL bioactivity in patients with macroprolactinaemia," Clinical Endocrinology, vol. 68, no. 1, pp. 72-76, 2008.

[33] N. Hattori, K. Ikekubo, T. Ishihara, K. Moridera, M. Hino, and H. Kurahachi, "Correlation of the antibody titers with serum prolactin levels and their clinical course in patients with antiprolactin autoantibody," European Journal of Endocrinology, vol. 130, no. 5, pp. 438-445, 1994.

[34] I. R. Wallace, N. Satti, C. H. Courtney et al., "Ten-year clinical follow-up of a cohort of 51 patients with macroprolactinemia establishes it as a benign variant," Journal of Clinical Endocrinology and Metabolism, vol. 95, no. 7, pp. 3268-3271, 2010.

[35] W. B. Malarkey, R. Jackson, and J. Wortsman, "Long-term assessment of patients with macroprolactinemia," Fertility and Sterility, vol. 50, no. 3, pp. 413-418, 1988.

[36] V. Leite, H. Cosby, L. G. Sobrinho, A. Fresnoza, M. A. Santos, and H. G. Friesen, "Characterization of big, big prolactin in patients with hyperprolactinaemia," Clinical Endocrinology, vol. 37, no. 4, pp. 365-372, 1992.

[37] A. Leaños-Miranda, D. Pascoe-Lira, K. A. Chávez-Rueda, and F. Blanco-Favela, "Detection of macroprolactinemia with the polyethylene glycol precipitation test in systemic lupus erythematosus patients with hyperprolactinemia," Lupus, vol. 10, no. 5, pp. 340-345, 2001.

[38] L. Kavanagh-Wright, T. P. Smith, J. Gibney, and T. J. McKenna, "Characterization of macroprolactin and assessment of markers of autoimmunity in macroprolactinaemic patients," Clinical Endocrinology, vol. 70, no. 4, pp. 599-605, 2009.

[39] N. Hattori, Y. Nakayama, K. Kitagawa, T. Ishihara, Y. Saiki, and C. Inagaki, "Anti-prolactin (PRL) autoantibody-binding sites (epitopes) on PRL molecule in macroprolactinemia," Journal of Endocrinology, vol. 190, no. 2, pp. 287-293, 2006.

[40] N. Hattori, K. Ikekubo, Y. Nakaya, K. Kitagawa, and C. Inagaki, "Immunoglobulin G subclasses and prolactin (PRL) isoforms in macroprolactinemia due to anti-PRL autoantibodies," Journal of Clinical Endocrinology and Metabolism, vol. 90, no. 5, pp. 3036-3044, 2005.

[41] N. Hattori, Y. Nakayama, K. Kitagawa, T. Li, and C. Inagaki, "Development of anti-PRL (prolactin) autoantibodies by homologous PRL in rats: a model for macroprolactinemia," Endocrinology, vol. 148, no. 5, pp. 2465-2470, 2007.

[42] A. Glezer, C. R. J. Soares, J. G. Vieira et al., "Human macroprolactin displays low biological activity via its homologous receptor in a new sensitive bioassay," Journal of Clinical Endocrinology and Metabolism, vol. 91, no. 3, pp. 1048-1055, 2006.

[43] A. Leaños-Miranda, G. Cárdenas-Mondragón, R. RiveraLeaños, A. Ulloa-Aguirre, and V. Goffin, "Application of new homologous in vitro bioassays for human lactogens to assess the actual bioactivity of human prolactin isoforms in hyperprolactinaemic patients," Clinical Endocrinology, vol. 65, no. 2, pp. 146-153, 2006.

[44] K. Teilum, J. C. Hoch, V. Goffin, S. Kinet, J. A. Martial, and B. B. Kragelund, "Solution structure of human prolactin," Journal of Molecular Biology, vol. 351, no. 4, pp. 810-823, 2005.

[45] P. Marrack, J. Kappler, and B. L. Kotzin, "Autoimmune disease: why and where it occurs," Nature Medicine, vol. 7, no. 8, pp. 899-905, 2001.

[46] M. Ohara, T. Hibi, N. Watanabe et al., "Immunoglobulin $\mathrm{G}$ subclass distribution of human anticolon antibodies in ulcerative colitis," Journal of Gastroenterology and Hepatology, vol. 10, no. 2, pp. 158-164, 1995.

[47] R. Guido, S. Valenti, L. Foppiani, D. De Martini, M. Cossu, and M. Giusti, "Prolactin decrease and shift to a normallike isoform profile during treatment with quinagolide in a patient affected by an invasive prolactinoma," Journal of Endocrinological Investigation, vol. 20, no. 5, pp. 289-293, 1997. 


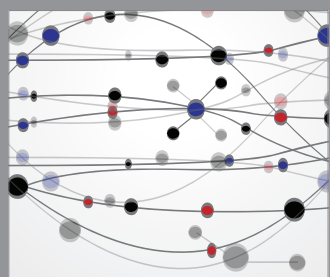

The Scientific World Journal
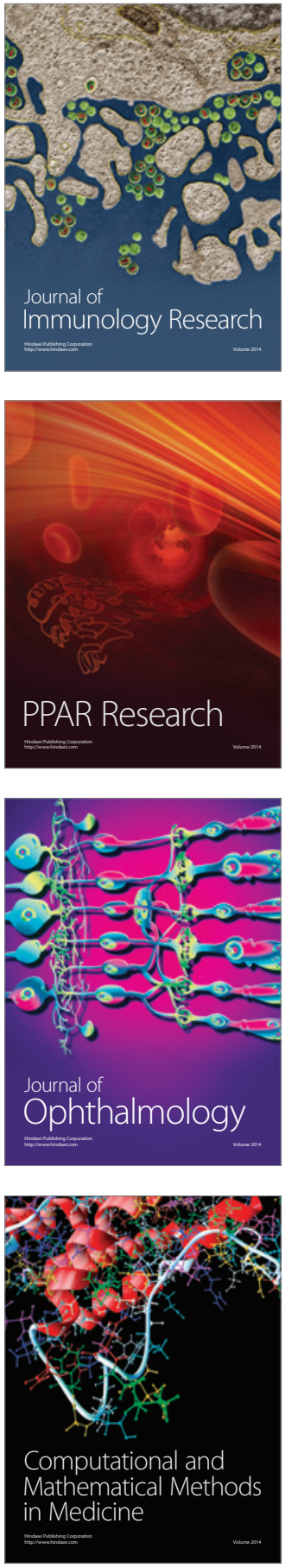

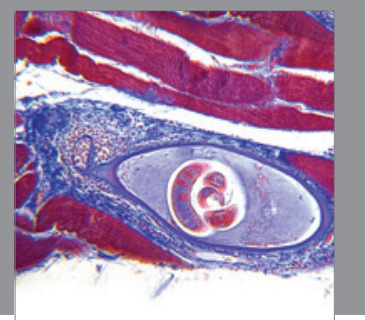

Gastroenterology

Research and Practice
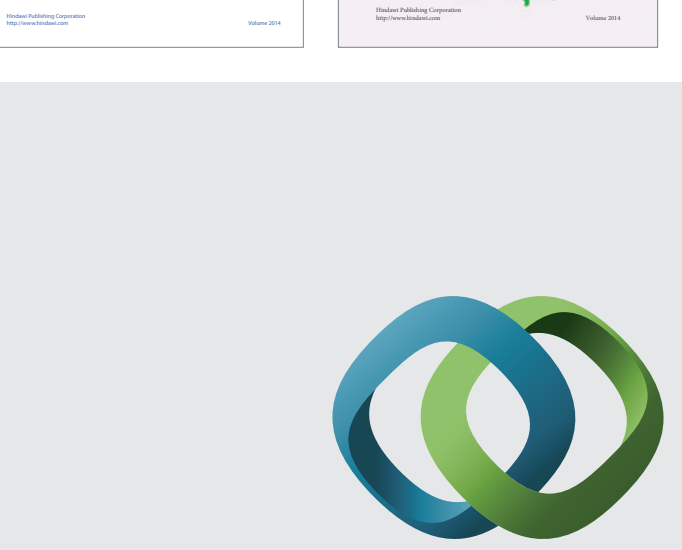

\section{Hindawi}

Submit your manuscripts at

http://www.hindawi.com
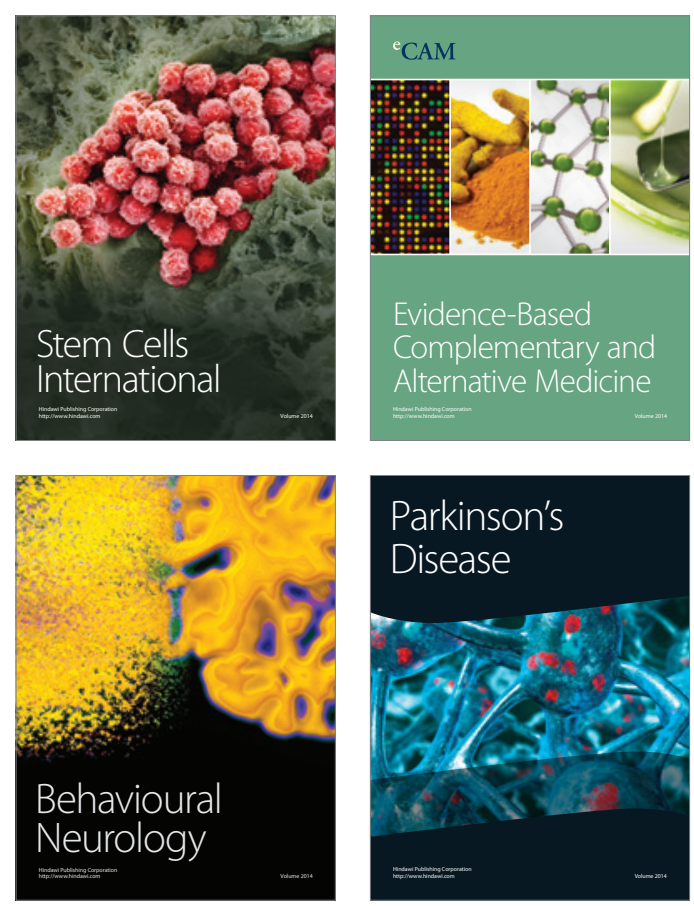

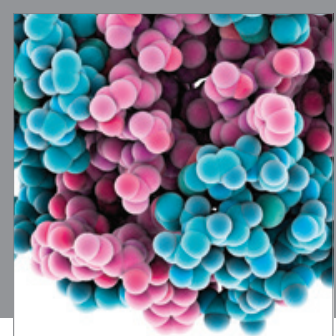

Journal of
Diabetes Research

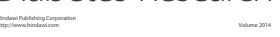

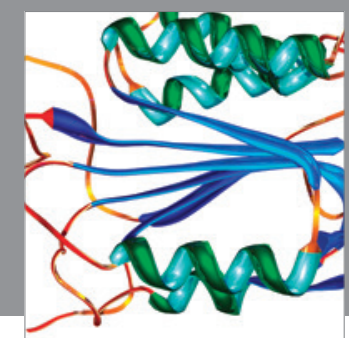

Disease Markers
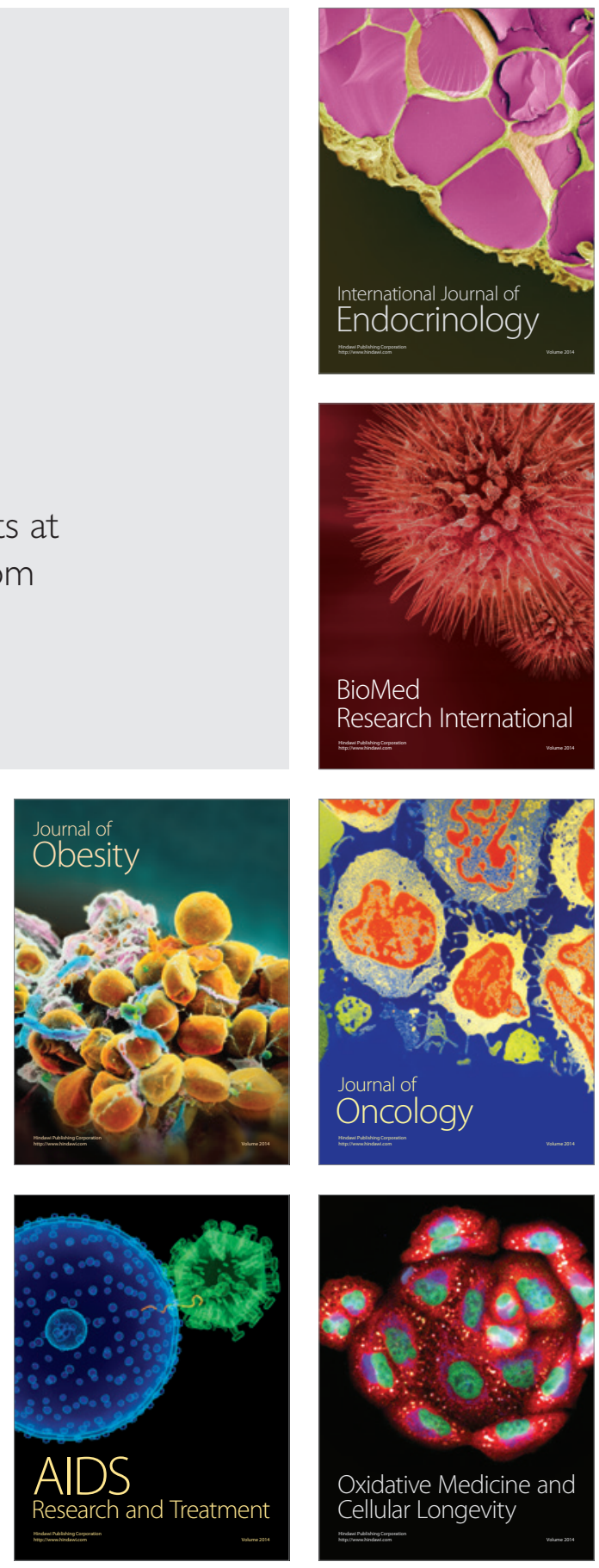